(C) Revista de Matemática: Teoría y Aplicaciones 2019 26(2) : 179-195

CIMPA - UCR ISSN: 1409-2433 (PRINT), 2215-3373 (ONLINE)

DOI: https://doi.org/10.15517/rmta.v26i2.33639

\title{
DEFINABLE GROUPS IN DCFA
}

\section{GRUPOS DEFINIBLES EN DCFA}

\author{
Ronald F. Bustamante-MedinA*
}

Received: 25/Jun/2018; Revised: 10/May/2019;

Accepted: 23/May/2019

Revista de Matemática: Teoría y Aplicaciones is licensed under a Creative Commons Reconocimiento-NoComercial-Compartirigual 4.0 International License.

Creado a partir de la obra en http://www.revistas.ucr.ac.cr/index.php/matematica

*Universidad de Costa Rica, Centro de Investigación en Matemática Pura y Aplicada (CIMPA) y Escuela de Matemática, San José, Costa Rica. E-Mail: ronald.bustamante@ucr.ac.cr 


\begin{abstract}
E. Hrushovski proved that the theory of difference-differential fields of characteristic zero has a model-companion. We denote it DCFA. In this paper we study definable abelian groups in a model of $D C F A$. First we prove that such a group is embeddable on an algebraic group. Then, we study one-basedeness, stability and stable embeddability of abelian definable groups.
\end{abstract}

Keywords: model theory of fields; supersimple theories; difference-differential fields; definable goups; abelian groups.

\title{
Resumen
}

E. Hrushovski demostró que la teoría de cuerpos diferenciables de diferencia de característica cero tiene una modelo-compañera. La denotamos DCFA. En este artículo estudiamos los grupos abelianos en un modelo de $D C F A$. Primero demostramos que tales grupos son isomorfos a un subgrupo de un grupo algebraico. Posteriormente, estudiaremos las propiedades de ser monobasados, estables y establemente inmersibles de grupos definibles abelianos.

Palabras clave: teoría de modelos de cuerpos; teorías supersimples; cuerpos diferenciales de diferencia; grupos definibles; grupos abelianos.

Mathematics Subject Classification: 11U09, 12H05, 12H10, 03C45, $03 \mathrm{C} 60$.

\section{Preliminaries}

The class of differentially closed fields of characteristic zero with a generic automorphism is elementary, we denote it DCFA.

Our aim in this paper is to study definable groups in models of $D C F A$ : in section 2, we prove that a definable group in a model of DCFA embeds in an algebraic group. In section 3, prove that we can reduce questions about 1-basedness and stable, stable embeddability in DCFA to questions about 1-basedness and stable, stable embeddability in either $D C F$ or $A C F A$. We use this, in section 4 , to study the model theory of definable abelian groups.

We give now a brief summary of what we know about DCFA. Since we will work in difference, differential and difference-differential fields we will denote the respective languages by $\mathcal{L}_{\sigma}, \mathcal{L}_{D}$ and $\mathcal{L}_{\sigma, D}$.

In [1], we give an axiomatization of $D C F A$ and prove its main properties: given a model of $D C F A$ it is of course a differentially closed field (model of $D C F$ ) and an algebraically closed field with a generic automorphism (model of $A C F A$ ). 
Independence is defined by linear disjointness. This theory is not complete, but its completions are easily described, those completions eliminate imaginaries (moreover, they satisfy the Independence Theorem over algebraically closes sets) and thus are supersimple and types are ranked by the $S U$-rank. Forking is determined by quantifier-free formulas, thus $D C F A$ is quantifier-free $\omega$-stable. A basis theorem for (perfect) difference-differential ideals imply that in a model of DCFA the difference-differential Zariski topology (defined in analogy with Zariski topology in algebraically closed fields) is Noetherian.

Let $(K, \sigma, D)$ be a model of $D C F A$, there are two important definable subfields of $K$, the field of constants $\mathcal{C}=\{x \in K: D x=0\}$ and the fixed field Fix $(\sigma)=\{x \in K: \sigma(x)=x\}$.

Given $a \in K$ and $A \subseteq K$, we define the $(\sigma, D)$-transcendence degree of $a$ over $A$ as the transcendence degree of the difference-differential field generated by $A$ and $a$ over $A$. In the cases of $D C F$ and $A C F A$ the finiteness of such a degree is equivalent to the finiteness of the rank of $a$ over $A$. However, this does not hold for DCFA: in [3], we give an example of a set whose generic type has infinite $(\sigma, D)$-transcendence degree but $S U$-rank 1 . This represents a difficulty in the treatment of definable groups, so we shall try different ways to describe definable groups departing from properties of groups definable in differential and difference fields.

In [2] and [4], we proved that Zilber's dichotomy holds for DCFA: a type of $S U$-rank 1 either has a simple geometry (it is 1-based) or has a strong interaction with (is non-orthogonal to) $\mathrm{Fix}(\sigma) \cap \mathcal{C}$.

We now introduce some definitions and useful facts about definable groups in supersimple theories. Let $T$ be a supersimple theory, $M$ a saturated model of $T$, let $G$ be a type-definable (definable by an infinite number of formulas) group and let $A \subset M$ be a set of parameters.

Definition 1 Let $p \in S(A)$. We say that $p$ is a left generic type of $G$ over $A$ if it is realized in $G$ and for every $a \in G$ and $b$ realizing $p$ such that $a \downarrow_{A} b$, we have $b \cdot a \downarrow_{A} a$. 
The following result is proved in [13].

\section{Fact 1}

1. Let $a, b \in G$. If $t p(a / A b)$ is left generic of $G$, then so is $t p(b \cdot a / A b)$.

2. Let $p \in S(A)$ be realized in $G, B=\operatorname{acl}(B) \supset A$, and $q \in S(B) a$ non-forking extension of $p$. Then $p$ is a generic of $G$ if and only if $q$ is a generic of $G$.

3. Let $t p(a / A)$ be generic of $G$; then so is $t p\left(a^{-1} / A\right)$.

4. There exists a generic type of $G$.

5. A type is left generic if and only if it is right generic.

The following fact is proved in [14, chapter 5].

Fact 2 Let $H$ a type-definable subgroup of $G$,

1. Let $p \in S(A)$, then $p$ is a generic of $G$ over $A$ if and only if $S U(G)=S U(p)$.

2. $S U(G)=S U(H)$ if and only if $[H: G]<\infty$.

3. $S U(H)+S U(G / H) \leq S U(G) \leq S U(H) \oplus S U(G / H)$.

\section{Every definable group embeds in an algebraic group}

We introduce $*$-definable groups in stable theories. Suppose that $T$ is a complete theory and $M$ a saturated model of $T$. A $*$-tuple is a tuple $\left(a_{i}\right)_{i \in I}$, where $I$ is an index set of cardinality less than the cardinality of $M$, and $a_{i} \in M^{e q}$, for all $i \in I$. Let $A \subset M$. A $*$-definable set is a collection of $*$-tuples, indexed by the same set of parameters $I$, which is the set of realizations of a partial type $p\left(x_{i}\right)_{i \in I}$ over $A$. A $*$-definable group is a group with $*$-definable domain and multiplication.

The following propositions are proved in [9]. Recall that the canonical base of a strong type $p, C b(p)$ is the set that is fixed pointwise by the automorphisms that fix $p$. 
Proposition 1 Let $T$ be a stable theory; $M$ a saturated model of $T$. Let $a, b, c, x, y, z$ be *-tuples of $M$ of length strictly less than the cardinal of $M$, such that:

1. $\operatorname{acl}(M, a, b)=\operatorname{acl}(M, a, c)=\operatorname{acl}(M, b, c)$.

2. $\operatorname{acl}(M, a, x)=\operatorname{acl}(M, a, y)$ and $C b(\operatorname{stp}(x, y / M, a))$ is interalgebraic with a over $M$.

3. As in 2, with $b, z, y$ in place of $a, x, y$.

4. As in 2, with $c, z, x$ in place of $a, x, y$.

5. Other than $\{a, b, c\},\{a, x, y\},\{b, z, y\},\{c, z, x\}$, any 3-element subset of $\{a, b, c, x, y, z\}$ is independent over $\mathcal{M}$.

Then there is a *-definable group $H$ defined over $M$ and $a^{\prime}, b^{\prime}, c^{\prime} \in H$ generic over $M$ such that a is interalgebraic with $a^{\prime}$ over $M, b$ is interalgebraic with $b^{\prime}$ over $M, c$ is interalgebraic with $c^{\prime}$ over $M$ and $a^{\prime} \cdot b^{\prime}=c^{\prime}$.

Proposition 2 Let $T$ be a simple theory; $M$ a saturated model of T. Let $G, H$ be type-definable groups, defined over $K \prec M$, and let $a, b, c \in G$ and $a^{\prime}, b^{\prime}, c^{\prime} \in H$ such that:

1. a, b are generic independent over $M$.

2. $a \cdot b=c$ and $a^{\prime} \cdot b^{\prime}=c^{\prime}$.

3. $a$ is interalgebraic with $a^{\prime}$ over $M, b$ is interalgebraic with $b^{\prime}$ over $M$ and $c$ is interalgebraic with $c^{\prime}$ over $M$.

Then there is a type-definable over $M$ subgroup $G_{1}$ of bounded index in $G$, and a type-definable over $M$ subgroup $H_{1}$ of $H$ and a type-definable over $M$ isomorphism $f$ between $G_{1} / N_{1}$ and $H_{1} / N_{2}$, where $N_{1}$ and $N_{2}$ are finite normal subgroups of $G_{1}$ and $H_{1}$ respectively.

Remark 1 If $T$ in Proposition 2 is supersimple and $G, H$ are definable, then we can choose $G_{1}$ definable of finite index in $G$ and $f$ definable.

The following result is proved in [7].

Proposition 3 Let $G$ be a $*$-definable group in a stable structure. Then there is a projective system of definable groups with inverse limit $G^{\prime}$, and $a *$-definable isomorphism between $G$ and $G^{\prime}$. 
In [12], the author proved that a $\mathcal{L}_{D}$-definable (definable in the language of differential fields) group in $D C F$ is essentially a differential algebraic group and that a definable group in $D C F$ virtually embeds in an algebraic group.

So, to prove that a definable group in DCFA embeds in an algebraic group we will show that it embeds in a $\mathcal{L}_{D}$-definable group.

Theorem 1 Let $(\mathcal{U}, \sigma, D)$ be a model of DCFA, $K \prec \mathcal{U}$ and $G$ a $K$-definable group. Then there is an $\mathcal{L}_{D}$-definable group $H$, a definable subgroup $G_{1}$ of $G$ of finite index, and a definable isomorphism between $G_{1} / N_{1}$ and $H_{1} / N_{2}$, where $H_{1}$ is a definable subgroup of $H(\mathcal{U}), N_{1}$ is a finite normal subgroup of $G_{1}$, and $N_{2}$ is a finite normal subgroup of $H_{1}$.

Proof. Let $a, b, y$ be generic independent elements of $G$ over $K$. Let $x=a \cdot y$, $z=b^{-1} \cdot y, c=a \cdot b$, so $x=c \cdot z$.

Let $\bar{a}=\left(\sigma^{j}(a): j \in \mathbb{Z}\right)$, and similarly for $\bar{b}, \bar{c}, \bar{x}, \bar{y}, \bar{z}$. Then, as the model-theoretic algebraic closure of a set is the differential-field-theoretic algebraic closure of the set closed by $\sigma$, working in $D C F, \bar{a}, \bar{b}, \bar{c}, \bar{x}, \bar{y}, \bar{z}$ satisfy the conditions of Proposition 1. Thus there is a $*-\mathcal{L}_{D}$-definable group $H$ over $K$, and generics $a^{*}, b^{*}, c^{*} \in H$ such that $a^{*}$ and $b^{*}$ are independent over $K$, $c^{*}=a^{*} \cdot b^{*}, \bar{a}$ is interalgebraic with $a^{*}$ over $K, \bar{b}$ is interalgebraic with $b^{*}$ over $K$ and $\bar{c}$ is interalgebraic with $c^{*}$ over $K$ (the interalgebraicity, independence and generics in the sense of $D C F$ ).

Since $D C F$ is $\omega$-stable, by Proposition $3, H$ is the inverse limit of $H_{i}, i \in \omega$, where the $H_{i}$ are $\mathcal{L}_{D}$-definable groups.

Let $\pi_{i}: H \longrightarrow H_{i}$ be the $i$-th canonical epimorphism. Let $a_{i}=\pi_{i}\left(a^{*}\right)$, $b_{i}=\pi_{i}\left(b^{*}\right)$ and $c_{i}=\pi_{i}\left(c^{*}\right)$. Then $a^{*}$ is interalgebraic with $\left(a_{i}\right)_{i \in \omega}$ over $K$, $b^{*}$ is interalgebraic with $\left(b_{i}\right)_{i \in \omega}$ over $K$ and $c^{*}$ is interalgebraic with $\left(c_{i}\right)_{i \in \omega}$ over $K$, all interalgebraicities in the sense of $D C F$.

Since for $i<j, a_{i} \in K\left(a_{j}\right), b_{i} \in K\left(b_{j}\right)$ and $c_{i} \in K\left(c_{j}\right)$, there is $i \in \omega$ such that $a$ is interalgebraic with $a_{i}$ over $K, b$ is interalgebraic with $b_{i}$ over $K$ and $\mathrm{t} c$ is interalgebraic with $c_{i}$ over $K$ in the sense of DCFA. So we can apply Proposition 2 to $a, b, c \in G$ and $a_{i}, b_{i}, c_{i} \in H_{i}$.

Corollary 1.1 Let $G$ be a definable group. Then there is an algebraic group $H$, a definable subgroup $G_{1}$ of $G$ of finite index, and a definable isomorphism between $G_{1} / N_{1}$ and $H_{1} / N_{2}$, where $H_{1}$ is a definable subgroup of $H(\mathcal{U}), N_{1}$ is a finite normal subgroup of $G_{1}$, and $N_{2}$ is a finite normal subgroup of $H_{1}$. 


\section{Stability, stable embeddability and 1-basedness}

In this section we discuss how to apply results from [5] to obtain similar results in models of DCFA. We also give a criterion for 1-basedness in DCFA.

We begin with general definitions and facts on supersimple theories.

$T$ will denote a supersimple theory which eliminates imaginaries. Let $M$ be a saturated model of $T$.

Let us recall that two types $p, q$ over $A \subseteq M$ are orthogonal, denoted $p \perp q$, if for every set $B \supseteq A$ and every realisations $a, b$ of $p$ and $q$ respectively, $a \downarrow_{B} b$.

\section{Definition 2}

1. Let $A \subset M$ and let $S$ be an $(\infty)$-definable set over $A$. We say that $S$ is 1 -based iffor every tuple a of $S$ and every $B \supseteq A$, $a$ and $B$ are independent over $\operatorname{acl}(A a) \cap \operatorname{acl}(B)$.

2. A type is 1-based if the set of its realizations is 1-based.

The following useful result is proved in [15].

\section{Proposition 4}

1. The union of 1-based sets is 1-based.

2. If tp $(a / A)$ and tp $(b / A a)$ are 1-based, so is tp $(a, b / A)$.

We introduce now stable, stably embedded types (also called fully stable types).

Definition 3 A (partial) type $p$ over a set $A$ is stable, stably embedded if whenever a realizes $p$ and $B \supset A$, then $t p(a / B)$ is definable. Equivalently, let $P$ denote the set of realizations of $p$. Then $p$ is stable, stably embedded if and only if for all set $S \cap P^{n}$ where $S$ is definable, there is a set $S^{\prime}$ definable with parameters from $P$ and such that $S^{\prime} \cap P^{n}=S \cap P^{n}$. 
The following result is proved in [5, Appendix].

Lemma 1 In a model of $A C F A$, if $t p_{A C F A}(b / A)$ and $t p_{A C F A}(a / A b)$ are stable, stably embedded, so is $\operatorname{tp}_{A C F A}(a, b / A)$.

Remark 2 In [5, section 4], a certain property of models of ACFA (called superficial stability) is isolated, and guarantees that certain types over algebraically closed sets are stationary, and therefore definable. It follows from model theoretic considerations that if $\operatorname{tp}_{A C F A}(a / A)$ is such that for any algebraically closed set $B$ containing $A \quad t p_{A C F A}(a / B)$ is stationary, then $t p_{A C F A}(a / A)$ is stable, stably embedded; and a stable, stably embedded type is stationary.

Lemma 2 Let $(K, \sigma)$ be a model of ACFA, $A=\operatorname{acl}_{\sigma}(A) \subset K$ and $a \in K$. Then $\operatorname{tp}(a / A)$ is stationary if and only if $\operatorname{tp}(a / A) \perp(\sigma(x)=x)$, where acl $\sigma$ denotes the model-theoretic algebraic closure in ACFA.

Proof. Indeed, write $S U(a / A)=\omega k+n$, and let $b \in \operatorname{acl}_{\sigma}(A a)$ be such that $S U(b / A)=n$. Then $t p(b / A) \perp(\sigma(x)=x)$ and, by [5, Thm 4.11], $\operatorname{tp}\left(\operatorname{acl}_{\sigma}(A b) / A\right)$ is stationary. If $c \in \operatorname{acl}_{\sigma}(A a)$ satisfies some non-trivial difference equation over $a c l_{\sigma}(A b)$ then $S U(c / A b)<\omega$ and therefore $c \in a c l_{\sigma}(A b)$. Hence, by [6, Thm 5.3], $t p\left(a / a c l_{\sigma}(A b)\right)$ is stationary, and therefore so is $\operatorname{tp}(a / A)$.

For the converse, there are independent realizations $a_{1}, \cdots, a_{n}$ of $t p(a / A)$, and elements $b_{1}, \cdots, b_{m} \in F i x(\sigma)$ such that $\left(a_{1}, \cdots, a_{n}\right)$ and $\left(b_{1}, \cdots, b_{m}\right)$ are not independent over $A$. Looking at the field of definition of the algebraic locus of $\left(b_{1}, \cdots, b_{m}\right)$ over $\operatorname{acl}_{\sigma}\left(A, a_{1}, \cdots, a_{n}\right)$, there is some $b \in \operatorname{Fix}(\sigma) \cap \operatorname{acl}_{\sigma}\left(A, a_{1}, \cdots, a_{n}\right), b \notin A$. Then $\operatorname{tp}(b / A)$ is not stationary: if $c \in \operatorname{Fix}(\sigma)$ is independent from $b$ over $A$, then $\operatorname{tp}(b / A)$ has two distinct non-forking extensions to $A c$, one in which $\sqrt{b+c} \in$ Fix $(\sigma)$, the other in which $\sqrt{b+c} \notin F i x(\sigma)$. Hence $t p\left(a_{1}, \cdots, a_{n} / A\right)$ is not stationary, and neither is $t p(a / A)$.

It is important to note that stationarity alone does not imply stability: if $a$ is transformally transcendental over $A=\operatorname{acl}_{\sigma}(A)$ (a is not the root of a non-zero $\sigma$-polynomial over $A$ ), then $\operatorname{tp}_{A C F A}(a / A)$ is stationary, but it is not stable.

These results can be used to give sufficient conditions on types in DCFA to be stationary, and stable, stably embedded. 
Proposition 5 Let $(K, \sigma, D)$ be a model of DCFA, let $A=\operatorname{acl}(A) \subset K$, and a a tuple in $K$.

1. Assume that $\operatorname{tp}_{A C F A}\left(a, D a, D^{2} a, \cdots / A\right) \perp \sigma(x)=x$. Then $t p(a / A)$ is stationary.

2. Assume that for every $n$, every extension of $t p_{A C F A}\left(D^{n} a / A a \cdots D^{n-1} a\right)$ is orthogonal to $(\sigma(x)=x)$. Then $t p(a / A)$ is stable, stably embedded. It is also 1-based.

3. If $\operatorname{tp}(a / A)$ has an extension that is not orthogonal to $(\sigma(x)=x)$, then $t p(a / A)$ is not stable, stably embedded.

\section{Proof.}

1. As $\operatorname{tp}_{A C F A}\left(a, D a, D^{2} a, \cdots / A\right) \perp \sigma(x)=x$, Lemma 2 implies that $t p_{A C F A}\left(a, D a, D^{2} a, \cdots / A\right)$ is stationary. Since the $t p(a / A)$ is determined by $t p_{A C F A}\left(a, D a, D^{2} a, \cdots / A\right), t p(a / A)$ is stationary: Let $b, c$ be two realizations of non-forking extensions of $t p(a / A)$ to a set $B=\operatorname{acl}(B) \supset A$. As $t_{A C F A}\left(a, D a, D^{2} a, \cdots / A\right)$ is stationary we have that $t p_{A C F A}\left(b, D b, D^{2} b, \cdots / B\right)=t p_{A C F A}\left(c, D c, D^{2} c, \cdots / B\right)$. If $\varphi(x)$ is an $\mathcal{L}_{\sigma, D}(B)$-formula satisfied by $b$, then there is a $\mathcal{L}_{\sigma}(B)$-formula $\psi\left(x_{0}, \cdots, x_{k}\right)$ such that $\phi(b)=\psi\left(b, D b, \cdots, D^{k} b\right)$; so we have $\psi\left(x_{0}, \cdots, x_{k}\right) \in t p_{A C F A}\left(b, D b, D^{2} b, \cdots / B\right)=t p_{A C F A}\left(c, D c, D^{2} c, \cdots / B\right)$. This implies that $t p(b / B)=t p(c / B)$, and thus $t p(a / A)$ is stationary.

2. By Lemma 2 for all $n \in \mathbb{N}$ and for all $B \supset A, t p_{A C F A}\left(D^{n} a / B a \cdots D^{n-1} a\right)$ is stationary. Thus, by Remark 2, for all $n, t p_{A C F A}\left(D^{n} a / A a \cdots D^{n-1} a\right)$ is stable, stably embedded and 1-based. By Lemma 1 stability, stable embeddability is preserved by extensions, hence $t p_{A C F A}(a, D a, \cdots / A)$ is stable, stably embedded, and this implies that all extensions to algebraically closed sets are stationary. As above, we deduce that all extensions of $t p(a / A)$ to algebraically closed sets are stationary, hence $t p(a / A)$ is stable, stably embedded. By Proposition 4 we have also that $t p_{A C F A}(a, D a, \cdots / A)$ is 1-based. As $t p(a / A)$ is determined by $t p_{A C F A}\left(a, D a, D^{2} a, \cdots / A\right), t p(a / A)$ is 1-based.

3. Let $B=\operatorname{acl}(B) \supset A$ such that $t p(a / B) \not \perp \sigma(x)=x$. If we repeat the "converse" part of the proof of Lemma 2 we conclude that $t p(a / A)$ is not stationart, thus it is not stable, stably embedded. 
Remark 3 Let $A, K$ and a be as above.

1. If $S U(a / A)=1$, then the stationarity of $\operatorname{tp}(a / A)$ implies its stability and stable embeddability.

2. There are examples of types of SU-rank 1 which satisfy Proposition 5(1) above but do not satisfy Proposition 5(2). Thus condition Proposition 5(2) is not implied by stationarity.

Corollary 1.2 Let $A=\operatorname{acl}(A)$, and a a tuple in $\mathcal{C}$. Then $t p(a / A)$ is stable, stably embedded if and only if $\operatorname{tp}_{A C F A}(a / A)$ is stable, stably embedded. In this case, it will also be 1-based.

Proposition 6 Let $A=\operatorname{acl}(A) \subset K$, and a a tuple in $K$, with $S U(a / A)=1$. If $\operatorname{tp}_{A C F A}(a / A) \perp(\sigma(x)=x)$ then $t p(a / A)$ is stable, stably embedded. In particular, if $\operatorname{tp}_{A C F A}(a / A)$ is stable, stably embedded, then so is $t p(a / A)$.

Proof. Suppose that $\operatorname{tp}(a / A)$ is not stable, stably embedded; then there is $B=\operatorname{acl}(B) \supset A$ such that $\operatorname{tp}(a / B)$ is not stationary, and therefore $\operatorname{tp}_{A C F A}\left(a, D a, D^{2} a, \ldots / B\right)$ is not stationary.

By Proposition $5 t p_{A C F A}\left(a, D a, D^{2} a, \ldots / A\right) \not \perp(\sigma(x)=x)$. Hence, there is some algebraically closed difference field $L$ containing $A$, which is linearly disjoint from $a_{A C F A}(A a)$ over $A$, and an element $b \in \operatorname{Fix}(\sigma) \cap(\operatorname{Lacl}(A a))^{a l g}, b \notin L$. Looking at the coefficients of the minimal polynomial of $b$ over $\operatorname{Lacl}(A a)$, we may assume that $b \in \operatorname{Lacl}(A a)$. Let $M=\operatorname{acl}(L)$, and chose $\left(M^{\prime}, L^{\prime}\right)$ realizing $t p(M, L / A)$ and independent from $a$ over $A$. Then $\operatorname{qftp}_{A C F A}\left(L^{\prime} / A a\right)=\operatorname{qftp}_{A C F A}(L / A a)$ and there is $b^{\prime} \in L^{\prime} a c l(A a)$ such that $\sigma\left(b^{\prime}\right)=b^{\prime}$. Since $S U\left(a / L^{\prime}\right)=1$, we get $a \in \operatorname{acl}\left(L^{\prime} b^{\prime}\right)=L\left(b^{\prime}\right)_{D}^{a l g}$. This implies that $t_{A C F A}\left(a / L^{\prime}\right) \not \perp(\sigma(x)=x)$, and gives us a contradiction.

Remark 4 As stated, the result of Proposition 6 is false if one only assumes $S U(a / A)<\omega$. The correct formulation in that case is as follows:

Assume $S U(a / A)<\omega$ and that acl ${ }_{\sigma}(A a)$ contains a sequence $a_{1}, \cdots, a_{n}$ of tuples such that, for all $i \leq n$, working in DCFA, $S U\left(a_{i} / A a_{1}, \cdots, a_{i-1}\right)=1$.

Under these hypotheses, if $\operatorname{tp}_{A C F A}(a / A)$ is stable, stably embedded then so is $\operatorname{tp}(a / A)$.

The proof of the following lemma is analogue to the last statement in the proof of Proposition 5(2). 
Lemma 3 Let a be a tuple of a model of DCFA, and A a subset of that model. If $t p_{D C F}(a / A)$ is 1-based then $\operatorname{tp}(a / A)$ is 1-based.

The [5, Lemma 2, Lemma 3] and Proposition 4 imply the following condition for 1-basedness, stability and stable embeddability for groups.

Theorem 2 Let $1 \longrightarrow G_{1} \longrightarrow G_{2} \longrightarrow G_{3} \longrightarrow 1$ be a short exact sequence of definable groups in a simple theory. Then $G_{2}$ is stable, stably embedded (resp. 1-based) if and only if $G_{1}$ and $G_{3}$ are stable, stably embedded (resp. 1-based).

\section{Abelian groups}

In this section, we study abelian groups defined over some subset $K=\operatorname{acl}(K)$ of a model $(\mathcal{U}, \sigma, D)$ of $D C F A$. We investigate whether they are 1-based, and whether they are stable, stably embedded.

In [3, Lemma 4.3] we proved that a definable subgroup of a connected differential algebraic group has finite index in its $(\sigma, D)$-Zariski closure. This, together with Corollary 1.1 and Theorem 2, implies that the study of definable abelian groups may be reduced to the case when the group $H$ is a quantifier-free definable subgroup of some commutative algebraic group $G$, and $G$ has no proper (infinite) algebraic subgroup, i.e. $G$ is either $\mathbb{G}_{a}, \mathbb{G}_{m}$, or a simple abelian variety $A$.

From now on we suppose all the groups are quantifier-free definable.

We study now all three cases for $G$.

\section{The additive group}

Proposition 7 No infinite definable subgroup of $\mathbb{G}_{a}^{n}(\mathcal{U})$ is 1-based.

Proof. Let $H<\mathbb{G}_{a}^{n}$ be a definable infinite group. By [3, Lemma 4.4], $H$ is quantifier-free definable and contains a definable subgroup $H_{0}$ which is definably isomorphic to $\operatorname{Fix}(\sigma) \cap \mathcal{C}$. Hence $H$ is not 1-based.

\section{The multiplicative group}

The logarithmic derivative $l D: \mathbb{G}_{m} \rightarrow \mathbb{G}_{a}, x \mapsto D x / x$ is a group epimorphism with $\operatorname{Ker}(l D)=\mathbb{G}_{m}(\mathcal{C})($ see $[11])$.

Given a polynomial $P(T)=\sum_{i=0}^{n} a_{i} T^{i} \in \mathbb{Z}[T]$, we denote by $P(\sigma)$ the homomorphism defined by $x \mapsto \sum_{i=0}^{n} a_{i} \sigma^{i}(x)$. 
Proposition 8 Let $H$ be a quantifier-free $\mathcal{L}_{\sigma, D}$-definable subgroup of $\mathbb{G}_{m}$. If $l D(H) \neq 0$ then $H$ is not 1-based. If $l D(H)=0$ then there is a polynomial $P(T)$ such that $H=\operatorname{Ker}(P(\sigma))$. Then we have that $H$ is 1-based if and only if $P(T)$ is relatively prime to all cyclotomic polynomials $T^{m}-1$ for all $m \in \mathbb{N}$.

Proof. By Proposition 7, if $l D(H) \neq 0$ then $H$ is not 1-based. If $l D(H)=0$, as $\operatorname{Ker}(l D)=\mathbb{G}_{m}(\mathcal{C}), H$ is $\mathcal{L}_{\sigma}$-definable in $\mathcal{C}$. Hence there is a polynomial $P(T)=\sum_{i=0}^{n} a_{i} T^{i} \in \mathbb{Z}[T]$ such that $H$ is defined by $\Pi_{i=0}^{n} \sigma^{i}\left(X^{a_{i}}\right)=1$. In $A C F A, H$ is 1-based, stable, stably embedded if and only if $P(T)$ is relatively prime to all cyclotomic polynomials $T^{m}-1$ for $m \geq 1$ (see [8]). By Proposition 5 the same holds for DCFA.

\section{Abelian varieties}

Definition 4 An abelian variety is a connected algebraic group A which is complete, that is, for any variety $V$ the projection $\pi: A \times V \rightarrow V$ is a closed map.

As a consequence of the definition we have that an abelian variety is commutative.

Let $B$ be an algebraic subgroup of an abelian variety $A$. Then $A / B$ is an abelian variety. If in addition $B$ is connected, $B$ is an abelian variety. An abelian variety is called simple if it has no infinite proper abelian subvarieties. Let $A$ and $B$ be two abelian varieties. Let $f: A \rightarrow B$ be a homomorphism. We say that $f$ is an isogeny if $f$ is surjective and $\operatorname{Ker}(f)$ is finite. We say that $A$ and $B$ are isogenous if there are isogenies $f: A \rightarrow B$ and $g: B \rightarrow A$.

Proposition 9 ([10]). There is no nontrivial algebraic homomorphism from a vector group into an abelian variety.

Now we mention some properties concerning 1-basedness of abelian varieties in difference and differential fields.

Consider a saturated model $(\mathcal{U}, \sigma)$ of $A C F A$. In [8], Hrushovski gives a full description of definable subgroups of $A(\mathcal{U})$ when $A$ is a simple abelian variety defined over $\mathcal{U}$. When $A$ is defined over $\operatorname{Fix}(\sigma)$, this description is particularly simple, at least up to commensurability. Let $R=\operatorname{End}(A)$ (the ring of algebraic endomorphisms of $A$ ). If $P(T)=\sum_{i=0}^{n} e_{i} T^{i} \in R[T]$, define $\operatorname{Ker}(P(\sigma))=\left\{a \in A(\mathcal{U}) \mid \sum_{i=0}^{n} e_{i}\left(\sigma^{i}(a)\right)=0\right\}$. 
Proposition 10 ([8]). Let $A$ be a simple abelian variety defined over $\mathcal{U}$, and let $B$ be a definable subgroup of $A(\mathcal{U})$ of finite $S U$-rank.

1. If $A$ is not isomorphic to an abelian variety defined over $(F i x(\sigma))^{\text {alg }}$, then $B$ is 1-based and stable, stably embedded.

2. Assume that $A$ is defined over Fix $(\sigma)$. Then there is $P(T) \in R[T]$ such that $B \cap \operatorname{Ker}(P(\sigma))$ has finite index in $B$ and in $\operatorname{Ker}(P(\sigma))$. Then $B$ is 1-based if and only if the polynomial $P(T)$ is relatively prime to all cyclotomic polynomials $T^{m}-1, m \in \mathbb{N}$. If $B$ is 1-based, then it is also stable, stably embedded.

We work now in a saturated model $(\mathcal{U}, D)$ of $D C F$. The following is proved in [11].

Proposition 11 Let $A$ be an abelian variety. Then there is a $\mathcal{L}_{D}$-definable (canonical) homomorphism $\mu: A \rightarrow \mathbb{G}_{a}^{n}$, for $n=\operatorname{dim}(A)$, such that $\operatorname{Ker}(\mu)$ has finite Morley rank (a generalization of the notion of algebraic dimension).

$\operatorname{Ker}(\mu)$, is known as the Manin kernel of $A$, we denote it by $A^{\sharp}$.

Proposition 12 (Properties of the Manin kernel, see [11] for the proofs). Let $A$ and $B$ be abelian varieties. Then,

1. $A^{\sharp}$ is the Kolchin closure of the torsion subgroup Tor $(A)$ of $A$.

2. $(A \times B)^{\sharp}=A^{\sharp} \times B^{\sharp}$, and if $B<A$ then $B \cap A^{\#}=B^{\#}$.

3. A differential isogeny between $A^{\sharp}$ and $B^{\sharp}$ is the restriction of an algebraic isogeny from $A$ to $B$.

We say that an abelian variety descends to the constants if it is isomorphic to an abelian variety defined over the constants.

Proposition 13 (DCF, see [11]). Let $A$ be a simple abelian variety. If $A$ is defined over $\mathcal{C}$, then $A^{\sharp}=A(\mathcal{C})$. If $A$ does not descend to the constants, then $A^{\sharp}$ is strongly minimal and 1-based. 
We now return to DCFA and fix a saturated model $(\mathcal{U}, \sigma, D)$ of $D C F A$ and a simple abelian variety $A$ defined over $K=\operatorname{acl}(K) \subset \mathcal{U}$.

Let $H$ be an $\mathcal{L}_{\sigma, D}$-definable connected subgroup of $A$ defined over the difference-differential field $K$ and let $\tilde{H}$ be its $(\sigma, D)$-Zariski closure. Since $H$ is 1-based if and only if $\tilde{H}$ is 1-based (see [3, Lemmas 4.3 and 4.4]), we can suppose that $H$ is quantifier-free definable and quantifier-free connected.

Let $\mu: A \rightarrow \mathbb{G}_{a}^{d}$ as in Proposition 11. If $H \not \subset K e r \mu$ then by Proposition 7 $H$ is not 1-based. Assume that $H \subset A^{\sharp}$.

We first show a very useful lemma.

Lemma 4 Let $H$ be a quantifier-free definable subgroup of $A^{\sharp}$ which is quantifier-free connected. Then $H=H^{\prime} \cap A^{\sharp}$ for some quantifier-free $\mathcal{L}_{\sigma}$-definable subgroup $H^{\prime}$ of $A$.

Proof. Our hypotheses imply that there is an integer $k$ and a differential subgroup $S$ of $A \times A^{\sigma} \times \cdots \times A^{\sigma^{k}}$, such that $H=\left\{a \in A:\left(a, \sigma(a), \cdots, \sigma^{k}(a)\right) \in S\right\}$. By Proposition 12.2, replacing $S$ by its Zariski closure $\bar{S}$ we get $H=\left\{a \in A^{\sharp}:\left(a, \sigma(a), \cdots, \sigma^{k}(a)\right) \in \bar{S}\right\}$. Thus $H=H^{\prime} \cap A^{\sharp}$, with $H^{\prime}=\left\{a \in A:\left(a, \sigma(a), \cdots, \sigma^{k}(a) \in \bar{S}\right\}\right.$.

Let us state an immediate consequence of Lemma 4:

Corollary 2.1 Iffor all $k \in \mathbb{N}, A$ and $A^{\sigma^{k}}$ are not isogenous, then $S U\left(A^{\sharp}\right)=1$.

Case1: $A$ is isomorphic to a simple abelian variety $A^{\prime}$ defined over $\mathcal{C}$.

We can suppose that $A$ is defined over $\mathcal{C}$. Then, by Proposition 13, $A^{\sharp}=A(\mathcal{C})$. Hence, by Proposition $5, H$ is 1 -based for DCFA if and only if it is 1-based for $A C F A$; and in that case, by Corollary 1.2, it will also be stable, stably embedded.

If $H=A(\mathcal{C})$ then we know that $H$ is not 1-based in $A C F A$.

If $H$ is a proper subgroup of $A(\mathcal{C})$, Proposition 10 gives a precise description of that case.

Case2: $A$ does not descend to $\mathcal{C}$.

Then, by [11, Section 5], $A^{\sharp}$ is strongly minimal and 1-based for $D C F$. By Lemma 3 it is 1-based for DCFA.

We will now investigate when $H$ is stable, stably embedded. By 1-basedness and quantifier-free $\omega$-stability, we know that if $X \subset A^{\sharp}$ is quantifier-free definable, then $X$ is a Boolean combination of cosets of quantifier-free definable subgroups of $A^{\sharp}$. 
Assume first that $H \neq A^{\sharp}$, and let $a$ be a generic of $H$ over $K$. Then $H$ is finite-dimensional, and therefore $S U(H)<\omega$. As $H$ is 1-based, there is an increasing sequence of subgroups $H_{i}$ of $H$ with $S U\left(H_{i+1} / H_{i}\right)=1$.

By Lemma 4, we may assume that $H_{i}=U_{i} \cap A^{\sharp}$ for some quantifier-free $\mathcal{L}_{\sigma}$-definable subgroups $U_{i}$ of $A$. Note that Lemma 4 also implies that each quotient $U_{i+1} / U_{i}$ is $c$-minimal (i.e., all quantifier-free definable $\mathcal{L}_{\sigma}$-definable subgroups are either finite or of finite index). Furthermore, by elimination of imaginaries in $A C F A, a_{\sigma}(K a)$ contains tuples $a_{i}$ coding the cosets $a+U_{i}$. Hence, $t p(a / K)$ satisfies the conditions of Remark 4 and we obtain that if $t p_{A C F A}(a / K)$ is stable, stably embedded then so is $t p(a / K)$.

For the other direction, observe that if $\operatorname{tp}_{A C F A}(a / K)$ is not stable, stably embedded, then for some $i$, the generic $A C F A$-type of $U_{i+1} / U_{i}$ is non-orthogonal to $\sigma(x)=x$, and there is a $\left(\mathcal{L}_{\sigma}\right)$-definable morphism $\psi$ with finite kernel $U_{i+1} / U_{i} \rightarrow B\left(F i x\left(\sigma^{k}\right)\right)$ for some $k$ and abelian variety $B$ (see [8]). But, returning to DCFA, no non-algebraic type realized in Fix $\left(\sigma^{k}\right)$ can be stable, stably embedded, since for instance the formula $\varphi(x, y)=\exists z z^{2}=x+y \wedge \sigma(z)=z$ is not definable (Proposition 5,3). This proves the other implication.

Thus we have shown:

If $H$ is finite dimensional, then $t p(a / K)$ is stable, stably embedded if and only if $\operatorname{tp}_{A C F A}(a / K)$ is stable, stably embedded.

Using Lemma 4, Proposition 10 gives us a full description of that case.

In particular, we then have that if $H$ is not stable, stably embedded, then $A$ is isomorphic to an abelian variety defined over $\operatorname{Fix}\left(\sigma^{k}\right)$ for some $k$.

Let us now assume that $H=A^{\sharp}$. Let $a$ be a generic of $H$ over $K$. Then $t p_{A C F A}\left(a, \cdots, D^{m} a / K\right)$ is the generic type of an algebraic variety $V$, and is therefore stationary (by [5, Corollaries 2.11]). Thus, using the finite dimensional case, if $A$ is not isomorphic to an abelian variety defined over $(F i x(\sigma))^{a l g}$, then $H$ is stable, stably embedded. If $A$ is isomorphic to a variety $B$ defined over $\operatorname{Fix}\left(\sigma^{k}\right)$, via an isomorphism $\psi$, then the subgroup $\psi^{-1}\left(\operatorname{Ker}\left(\sigma^{k}-1\right)\right) \cap A^{\sharp}$ is not stable, stably embedded.

We summarize the results obtained. 
Theorem 3 Let $A$ be a simple abelian variety, and let $H$ be a quantifier-free definable subgroup of $A(\mathcal{U})$ defined over $K=\operatorname{acl}(K)$. If $H \not \subset A^{\sharp}(\mathcal{U})$, then $H$ is not 1-based. Assume now that $H \subset A^{\sharp}(\mathcal{U})$, and let a be a generic of $H$ over $K$. Then,

1. If $A$ is defined over the field $\mathcal{C}$ of constants, then $H$ is 1-based if and only if it is stable, stably embedded, if and only if every extensions of $t p_{A C F A}(a / K)$ is orthogonal to $(\sigma(x)=x)$. The results in [8] yield a complete description of the subgroups $H$ which are not 1-based.

2. If $A$ does not descend to the field $\mathcal{C}$ of constants, then $H$ is 1-based. Moreover,

(a) If $A$ is not isomorphic to an abelian variety defined over Fix $\left(\sigma^{k}\right)$ for some $k$, then $H$ is stable, stably embedded.

(b) Assume that $A$ is defined over Fix $(\sigma)$. Then $H$ is stable, stably embedded if and only if $\operatorname{tp}_{A C F A}(a / K)$ is stable, stably embedded. Again, the results in [8] give a full description of this case.

\section{Acknowledgements}

The author acknowledges support from the School of Mathematics and the Research Center of Pure and Applied Mathematics (CIMPA), project 821-B9-092, University of Costa Rica.

\section{References}

[1] R. Bustamante, Differentially closed fields of characteristic zero with a generic automorphism, Revista de Matemática: Teoría y Aplicaciones 14 (2007), no. 1, 81-100.

[2] R. Bustamante, Algebraic jet spaces and Zilber's dichotomy in DCFA, Revista de Matemática: Teoría y Aplicaciones 17 (2010), no. 1, 1-12.

[3] R. Bustamante, Rank and dimension in difference-differential fields, Notre Dame Journal of Formal Logic 52 (2011), no. 4, 403-414.

[4] R. Bustamante, Arc spaces and Zilber's dichotomy in dcfa, submitted, 2019.

[5] Z. Chatzidakis, E. Hrushovski, Model theory of difference fields, Trans. Amer. Math. Soc. 351 (1999), no. 8, 2997-3071. 
[6] Z. Chatzidakis, A. Pillay, Generic structures and simple theories, Ann. Pure Appl. Logic 95 (1998), no. 1-3, 71-92.

[7] E. Hrushovski, Unidimensional theories are superstable, Ann. Pure Appl. Logic 50 (1990), no. 2, 117-137.

[8] E. Hrushovski, The Manin-Mumford conjecture and the model theory of difference fields, Ann. Pure Appl. Logic 112 (2001), no. 1, 43-115.

[9] P. Kowalski, A. Pillay, A note on groups definable in difference fields, Proc. Amer. Math. Soc. 130 (2001), no. 1, 205-212.

[10] S. Lang, Abelian Varieties, reprint of the 1959 original, Springer, New York, 1983.

[11] D. Marker, Manin kernels, Connections between model theory and algebraic and analytic geometry, Vol. 6, Dipartimento di Matematica della Seconda Univ. di Napoli, Caserta, 2000.

[12] A. Pillay, Some foundational questions concerning differential algebraic groups, Pacific J. Math. 179 (1997), no. 1, 179-200.

[13] A. Pillay, Definability and definable groups in simple theories, J. Symbolic Logic 63 (1998), no. 3, 788-796.

[14] F. O. Wagner, Simple Theories, Kluwer Academic Publishers, Dordrecht, 2000.

[15] F. O. Wagner, Some remarks on one-basedness, J. Symbolic Logic 69 (2004), no. 1, 34-38. 
